\section{Regards sur l'économie allemande}

Bulletin économique du CIRAC

$67 \mid 2004$

Varia

\title{
Mutation du « modèle rhénan » et avenir du modèle européen
}

Gabriel Colletis

\section{OpenEdition}

\section{Journals}

Édition électronique

URL : http://journals.openedition.org/rea/3793

DOI : $10.4000 /$ rea.3793

ISBN : 978-2-8218-0830-0

ISSN : 1965-0787

\section{Éditeur}

CIRAC

Édition imprimée

Date de publication : 1 juillet 2004

Pagination : 7-14

ISSN : 1156-8992

\section{Référence électronique}

Gabriel Colletis, « Mutation du « modèle rhénan » et avenir du modèle européen », Regards sur l'économie allemande [En ligne], 67 | juillet 2004, mis en ligne le 08 octobre 2009, consulté le 01 mai 2019. URL : http://journals.openedition.org/rea/3793 ; DOI : 10.4000/rea.3793 


\section{Mutation du " modèle rhénan " et avenir du modèle européen}

\section{Gabriel Colletis}

Le débat sur la diversité versus la convergence des capitalismes, ou encore celui, plus limité peut-être, mais voisin, du choix d'un modèle socio-économique pour l'Europe, sont largement influencés par le devenir des compromis en cours d'élaboration tant au niveau européen (adoption d'une Constitution) qu'à celui des différents pays qui composent l'Europe élargie.

L'interprétation des transformations qui s'opèrent depuis quelque temps en Allemagne est essentielle à plus d'un titre. D'abord en raison de la puissance économique de ce pays, puissance renforcée par le récent élargissement au cœur duquel elle tient une place privilégiée. Mais aussi parce que le "modèle social " allemand, souvent désigné comme celui d'une "économie sociale de marché ", peut - avec celui, proche, proposé par la Suède et plus largement les pays scandinaves - se poser en rival du modèle dit " anglo-saxon ».

L'interprétation de ces mutations n'est pas une tâche facile parce que celles-ci sont en cours, mais aussi et surtout parce que s'affrontent, parfois violemment, des représentations divergentes de ce vers quoi ces mutations devraient tendre: pour les uns, une mutation radicale afin de permettre à l'économie et aux entreprises allemandes de s'insérer efficacement dans le processus de mondialisation, condition posée comme préalable du maintien d'un niveau de bien-être élevé ; pour les autres, une évolution qui préserve l'essence d'un modèle intégrant sans les hiérarchiser l'économique et le social.

Il y a un an environ, des commentateurs de toutes sortes évoquaient «l'enlisement » de l'Allemagne, son " essoufflement », ou encore le risque de "contamination » de l'Europe par une économie mal en point. Nombreux étaient également les économistes à agiter le spectre d'une déflation, posée comme risque "récurrent» pour l'Allemagne. Les raisons invoquées d'une telle situation, outre les difficultés spécifiques associées à l'unification, allaient alors de salaires excessifs ayant des conséquences négatives sur la demande intérieure et l'emploi, à la surévaluation du mark au moment de son entrée dans l'euro, en passant par l'absence de politiques 'd'envergure', ou encore l'incapacité des Allemands à s'adapter aux mutations: mondialisation et concurrence accrue, nouvelles technologies ou, dans une formulation plus récente et peut-être plus intéressante, l'entrée dans une économie fondée sur la connaissance.

Si ces différentes causes, avancées comme à l'origine des maux dont souffrirait l'Allemagne, ne sont pas indépendantes les unes des autres, c'est sans doute la dernière qui résume le mieux le sentiment d'une société susceptible de risquer le déclin car refusant d'évoluer, de se transformer pour tenir compte d'une nouvelle donne basée sur la mondialisation et les nouvelles technologies. Cette nouvelle donne exigerait ainsi de profondes réformes dans différents domaines, mais en premier lieu dans tout ce qui touche le rapport salarial : déterminants de la fixation et de l'évolution des salaires, réforme du marché du travail, 'allègement' d'un Etat-providence désormais considéré comme un fardeau.

Annoncées en mars 2003 sous la forme d'un document d'ensemble appelé Agenda 2010, les réformes sont immédiatement saluées comme « décrispant »

De l'Allemagne 'boulet' de la croissance en Europe..

... à l'Allemagne moteur de réformes « indispensables »? 
le débat économique européen (Le Monde, 10-07-2003). A peine quatre mois après leur annonce, elles semblent porter leurs premiers fruits avec l'amélioration qu'on leur prête des indices économiques. Et les commentateurs qui, quelques semaines auparavant, n'avaient de mots assez durs pour dénoncer les risques de déflation et "d'infection » de l'Europe par un pays rétif au changement, de louer un chancelier " courageux », réussissant à " briser les tabous ", d'abord au sein de son propre camp. L'essentiel, pour l'Allemagne comme pour l'Europe, aurait ainsi été de commencer à bouger (Le Monde, 27-07-2003).

\section{Les compromis institutionnalisés au fondement du capitalisme rhénan}

Capitalisme rhénan : des compromis institutionnalisés

Rapport salarial : compromis entre travail et capital

Entreprise : fonctionnement en partenariat et marché organisé

Un acteur public partie prenante de la société civile
Avant d'évoquer les changements survenus et leur interprétation, il convient d'indiquer sommairement quels sont les traits fondamentaux, selon nous, d'un capitalisme original, cherchant à combiner les intérêts du travail et ceux du capital dans les différents espaces de compromis que constituent les formes institutionnelles de ce capitalisme. Toute formation sociale (locale ou nationale) se caractérise d'abord, en effet, par un ensemble de compromis institutionnalisés qui peuvent être déclinés selon différents espaces de réalisation. Si on peut distinguer différentes formes économiques, sociales et politiques constitutives du « modèle rhénan » (Uterwedde, 2002), on peut aussi les combiner en les croisant autour de quelques traits essentiels ou espaces de compromis.

S'agissant de compromis entre le travail et le capital, le premier espace de compromis concerne à l'évidence le rapport salarial. Les éléments clés du rapport salarial caractérisant le capitalisme allemand sont : un haut niveau de productivité obtenu moins par intensification du travail que par un haut niveau de qualification, le rôle de l'apprentissage et de la promotion interne, l'importance des conventions collectives de branche et l'autonomie tarifaire, la co-détermination, les formes de flexibilisation négociées. Au final, l'objectif de stakeholder value l'emporte sur celui de shareholder value, alors qu'au niveau de la société dans son ensemble, solidarité et équité constituent le corollaire de la liberté individuelle, qui rime avec individualité - mais non avec individualisme, la liberté impliquant l'acceptation d'un certain nombre d'obligations sociales (Dreyfus, 2002).

L'entreprise est le lieu privilégié de réalisation du rapport salarial. Recherchant un compromis en interne, l'entreprise allemande 'classique' revêt en externe un fonctionnement partenarial ou en réseau. Souvent à caractère familial, même pour les plus importantes, du moins à l'origine, les entreprises allemandes, tout en n'ignorant pas les contraintes de concurrence, tentent de multiples manières de compléter le marché, voire parfois de le mettre entre parenthèses. Si la caricature de ce comportement a pu être autrefois la formation dans certains secteurs de «cartels », l'expression la plus habituelle aujourd'hui est celle d'un fonctionnement, si ce n'est concerté, du moins organisé des marchés, visant à éviter la concurrence sauvage et à favoriser les alliances, notamment entre PME, et entre PME et grands groupes ; ce, le plus souvent, au niveau local ou à celui d'une branche. On observera ici l'effet d'ancrage que ces alliances et réseaux produisent, le renforcement de la base productive allemande étant privilégié plutôt que le développement d'une base productive à l'étranger (Lallement). Enfin, il convient de rappeler qu'en dépit d'évolutions récentes, le système bancaire reste très impliqué de différentes façons dans le devenir des entreprises allemandes (voir REA 66/04).

L'acteur public allemand est un acteur collectif. Ceci va bien au-delà de la seule observation d'une organisation à caractère fédéral et s'étend aux relations entre l'acteur (les acteurs) public(s) et la société civile, y compris lorsque celles-ci s'organisent autour d'objectifs à caractère économique. Loin d'être extérieur ou 'au dessus' de la société civile, l'acteur public allemand est partie prenante de la société civile. Sa légitimité lui permet de jouer son rôle fondamental, non d'or- 
ganisateur et encore moins de superviseur, mais d'instance de validation de normes produites par la société civile elle-même. A ce titre, et s'agissant d'activités économiques, il est sans doute pertinent de considérer que les marchés en Allemagne sont des marchés «institués politiquement » et soumis à une « régulation sociétale » (Streeck, 1996). Les termes clés du fonctionnement de l'acteur public allemand peuvent se résumer à travers les notions de partage et délégation du pouvoir, de fédéralisme coopératif, et de subsidiarité.

Les compromis institutionnalisés élaborés dans les trois espaces interdépendants, celui du rapport salarial (se jouant, en grande partie, dans l'entreprise mais ne se limitant pas à ce seul espace puisqu'il implique d'autres dimensions comme le sens et l'organisation du système éducatif par exemple), celui du fonctionnement de l'entreprise, celui, enfin, du rapport de l'acteur public à la société civile, doivent non seulement être cohérents entre eux, mais encore être en cohérence avec la façon dont l'Allemagne, comme formation sociale nationale, s'insère dans le 'régime international'.

La cohérence externe du modèle allemand de capitalisme a reposé, au lendemain de la guerre et jusque dans les années 70 , sur une importante sousévaluation du deutsche mark. Celle-ci permettait, entre autres effets recherchés, de stimuler les exportations et de consolider certains des pôles de compétitivité de l'industrie allemande. La persistance de l'influence des idées de F. List en termes de " protectionnisme éducateur " n'est sans doute pas à négliger pendant cette période. On rappellera que $\mathrm{F}$. List a écrit son principal ouvrage, Le Système National d'Economie Politique, en 1841, à l'époque du grand débat sur l'Union douanière. Selon F. List, " le libre-échange est l'objectif, le protectionnisme, la voie pour y parvenir ». Le protectionnisme de List est, cependant, un protectionnisme provisoire et partiel. II ne s'applique qu'aux seules industries "dans l'enfance ». Ou, par extension, aux activités en nécessaire mutation, du fait, par exemple, d'une altération des conditions de marché ou du changement technologique.

De manière plus large cependant, la croissance économique allemande depuis le milieu des années 50 et jusqu'à nos jours s'apparente largement au schéma décrit par certains économistes ( $N$. Kaldor, notamment) d'une croissance « tirée » par les exportations. Un tel schéma suppose que le partage des gains de productivité s'effectue non pas seulement principalement entre salariés et détenteurs du capital (comme en France) mais entre ces deux premiers et les acheteurs extérieurs de produits allemands (sous la forme d'une baisse des prix relatifs à l'exportation). Par ailleurs, il convient d'observer que les producteurs allemands, à la différence de leurs homologues français et américains, mais à l'instar des producteurs japonais, privilégient le renforcement de leur base productive nationale, ce qui explique en partie la relative faiblesse de l'investissement direct allemand sortant (Lallement ; Commissariat Général du Plan, 1999).

II est cependant probable, notamment avec l'élargissement de l'UE, que le fort recouvrement entre la base productive des groupes allemands et le territoire national sera conduit à évoluer dans les prochaines années. Cette question sous-tend les évolutions du débat sur l'avenir du Standort Deutschland. En Allemagne, comme dans la plupart des pays développés, ce débat semble avoir glissé d'une problématique en termes de « compétitivité » à une interrogation sur « l'attractivité » du site. C'est sans doute ce glissement qui constitue la véritable signification actuelle, en lien avec la volatilité des flux financiers, de la mondialisation. Alors que la compétitivité renvoie aux échanges entre des économies nationales ouvertes entre elles (internationalisation), l'attractivité renvoie à la mobilité non des produits ou services, mais des facteurs : classiquement le capital et le travail (mondialisation). On observera que cette mobilité est fort différente selon qu'il s'agit du capital financier (volatile), du capital industriel ou productif, ainsi que du travail qualifié (nomades), ou du travail peu qualifié (de moins en moins mobile, du moins 'légalement', les délocalisations se
Des compromis cohérents avec une insertion internationale..

....initiée au lendemain de la guerre sur la base d'un DM sous-évalué..

... et d'un partage des gains de productivité intérieur / extérieur

Mais les mutations du contexte feront évoluer ce modèle... 
....alors que se pose la question du devenir du capitalisme français substituant à sa mobilité). II y a tout lieu de penser que, de plus en plus, la rémunération de ces différents 'facteurs' sera fonction de leur propension ou de leur capacité à se déplacer...

Ce glissement concerne les firmes les plus susceptibles de mobilité, c'est-àdire, pour l'essentiel, les firmes dites 'globales' : de grande ou très grande taille. On peut cependant estimer qu'il existe un lien entre l'ouverture du capital de ces firmes à des capitaux non résidents et leur propension à redéployer leur base productive (notamment par croissance externe) au niveau mondial. De ce point de vue, l'asymétrie de situation entre les groupes cotés français et leurs homologues allemands est éclatante : alors que les groupes allemands (comme ceux des autres pays développés : Etats-Unis, Royaume-Uni, etc.) restent aujourd'hui, pour l'essentiel, sous contrôle résident, les groupes français (surtout ceux du CAC 40) sont largement sous influence de capitaux non résidents, notamment nord-américains (Commissariat Général du Plan, 2004). Si on peut toujours se féliciter de l'attractivité des entreprises françaises pour les investisseurs étrangers, on peut en revanche se demander ce qu'il en est de l'influence que ces derniers sont susceptibles d'exercer sur les décisions stratégiques des premières. Au-delà encore, la question mérite d'être posée du devenir du capitalisme français comme tel (Commissariat Général du Plan, 1999).

\section{Agenda 2010 : une tentative de refondation du rapport salarial...}

Parmi les compromis institutionnalisés au fondement du capitalisme rhénan, le rapport salarial subit une mutation qui se joue, en grande partie, à travers l'Agenda 2010. Observons, cependant, que les autres espaces de compromis (fonctionnement de l'entreprise, mode d'action de l'acteur public, adhésion au régime international ou mondial) sont directement interpellés par les mutations du rapport salarial, reflet de la cohérence des compromis institutionnels.

De vives réactions...

L'annonce des réformes contenues dans le document Agenda 2010 a suscité de vives réactions, tant en Allemagne qu'à l'étranger, et en Europe singulièrement. L'Allemagne semble, avec cet ensemble de réformes, rompre avec l'immobilisme qui lui est fréquemment reproché. Immobilisme parfois imputé à ce qui serait devenu, selon certains, un culte plus qu'une culture du consensus. II serait ainsi devenu grand temps pour l'Allemagne de s'attaquer enfin aux obstacles qui freineraient structurellement l'activité - le coût et le droit du travail, les retraites et la fiscalité -, seul moyen de 'vraiment' relancer la croissance.

Pour ce faire, il s'agirait de « rompre avec tous les acquis sociaux facteurs d'immobilisme, qu'ils soient le fait des syndicats, du patronat ou de l'exception artisanale». Les réformes à mettre en œuvre devraient viser la baisse des prélèvements, «déplacer le curseur de l'effort collectif vers l'effort individuel» (Uterwedde, 2003). Selon J. Ackermann, président de la Deutsche Bank, " le changement éveille [chez les Allemands] la peur de perdre quelque chose de familier [...]. Cela se reflète dans les institutions orientées vers la construction de consensus. Utile dans les années d'après-guerre pour établir solidement la démocratie parlementaire, ce système s'avère inflexible et dépassé dans le contexte de changements rapides et de compétition globale » (Le Monde, 17-03-2003).

Rénover la production des normes...

Au-delà du contenu même des réformes, c'est donc la façon de produire les normes, c'est-à-dire le mode et la place des institutions formelles et informelles qui est en cause ou en jeu. «Modernisation » pour les uns, « démantèlement » pour les autres, la mutation du rapport salarial s'effectue dans un contexte culturel emprunt de tensions. La production des compromis est posée comme nécessitant un temps trop long à un moment où les mutations devraient se faire à un rythme rapide qui serait imposé par la mondialisation et l'accélération des échanges liés aux nouvelles technologies. Désormais, tout retard ou ralentisse- 
ment du rythme de mise en œuvre des réformes aurait comme tribut une capacité diminuée à innover (G. Schröder, Le Monde, 14 mai 2004).

Deux questions différentes, même si étroitement liées, sont donc posées : celle du contenu des réformes, et celle de la façon dont ces réformes sont produites, donc du mode de production des normes en Allemagne. Cette seconde question, parfois sous-estimée, du moins par les économistes, est aussi essentielle, nous semble-t-il, que la première. Ce qui pourrait se jouer ici concerne, en effet, une mutation profonde du système de valeurs sur lequel se fonde le capitalisme rhénan. Ce qui jusqu'alors était désigné comme relevant de la stabilité est désormais qualifié de facteur d'immobilisme : la production de consensus relèverait, dès lors, de la contrainte et ne serait plus considérée comme synonyme d'un nécessaire compromis, lui-même garant de prospérité économique et de bien-être social.

Susceptibles de s'émanciper des contraintes qu'imposerait un dispositif de régulation - par définition - trop contraignant, les (grandes) entreprises, principal acteur collectif porteur de cette mutation, poussent ainsi à cette mutation, et, plus concrètement, à l'allègement, voire parfois à la suppression des obstacles dits structurels. Ainsi, à titre d'illustration, un marché du travail réglementé, autrefois synonyme de protection des salariés, mais aussi garde-fou du jeu concurrentiel visant à éviter une concurrence par les coûts salariaux, est présenté aujourd'hui comme une contrainte dont l'impossibilité de contournement ou d'atténuation aurait comme conséquence la délocalisation des activités.

Le contenu des réformes dont le sens est celui d'une mutation en profondeur du rapport salarial est bien connu : il s'agit tout à la fois, de manière cohérente, de réformer le marché du travail en introduisant une dose importante de déréglementation, de comprimer le coût du système de santé, de réformer le système de retraite en introduisant une part de capitalisation. A côté de cela est envisagée une réforme fiscale dont le double objet est la simplification du système d'imposition et la réduction des taux.

L'esprit comme la lettre des réformes engagées est clair et se décline en termes de «sacrifices » demandés à la «société » (et pas seulement aux salariés, même si ceux-ci sont le plus fortement mis à contribution). Ces "sacrifices » visent à alléger le coût (surtout indirect) du travail, à assouplir la réglementation en matière de contrat de travail, à produire des économies sur le fonctionnement du système de protection sociale. Les accords salariaux de branche étant susceptibles d'être suspendus pour celles des entreprises qui seraient en difficulté et ne pourraient en sortir en raison d'un coût trop élevé pour elles de la main-d'œuvre, c'est un espace central de régulation des compromis qui est également remis en question, fût-ce en partie.

\section{...inspirée par une certaine représentation des mutations qu'imposerait un nouveau modèle de capitalisme}

Si nul ne conteste que les réformes engagées constituent des « sacrifices » et non des avancées sociales, c'est l'interprétation du sens de ces évolutions qui est en débat. Un même motif de mutation peut le plus souvent aussi bien servir la thèse de ceux qui estiment que ce sens est celui d'un démantèlement du système social, et la thèse, inverse, de ceux qui pensent qu'une modernisation est inévitable et que ces réformes sont indispensables pour préserver ce qui peut l'être de ce système. A titre d'illustration, le vieillissement de la population ou encore les progrès scientifiques dans le domaine médical peuvent aussi bien alimenter la thèse qu'il n'est ni possible ni souhaitable de réduire les budgets consacrés à la retraite et à la santé, que la thèse bien différente de la nécessaire introduction d'une part de capitalisation dans le système de retraite, ou encore celle de l'utilité d'une concurrence accrue dans l'offre et la gestion de l'offre de soins. II n'est ainsi, selon nous, pas possible de considérer qu'une des
... implique la mutation du système des valeurs

Les grandes entreprises poussent au changement

La déréglementation du marché du travail ...

... touche à un espace central de régulation des compromis
En débat : l'interprétation du sens de ces évolutions 
Un choc de représentations...

... lié à la nouvelle division cognitive du travai

Un nouveau bloc social dominant? Finance..

... groupes internationalisés...

... et adeptes du capitalisme libéral

Le modèle social allemand, un obstacle à la 'globalisation'? thèses en présence serait 'pragmatique' alors que l'autre ou toute autre serait 'idéologique'. Les thèses en présence relèvent, en fait, de points de vue différents ou, mieux : de systèmes de représentation différents.

La compréhension du débat prend un autre sens si, plutôt que d'opposer conservateurs et modernistes, idéologues et tenants du pragmatisme, on accepte qu'il s'agit d'un choc de représentations. De tels chocs se produisent toujours lorsqu'un 'bloc social' dominant, le plus souvent fondé sur une alliance, se fissure ou est contesté par un autre bloc en formation. Davantage qu'ailleurs en Europe, le bloc social dominant en Allemagne depuis la seconde moitié du XIX siècle était organisé autour de l'industrie manufacturière. Ce bloc comprenait deux acteurs collectifs principaux: le patronat industriel (s'appuyant sur les banques) et les syndicats.

C'est l'organisation de ce bloc qui est aujourd'hui altérée, mais non nécessairement remise en cause. Le ferment de cette altération réside moins dans les nouvelles technologies en elles-mêmes que dans la signification du processus de passage d'une division technique à une division cognitive du travail. II réside moins dans la progression de la valeur immatérielle des produits que dans la propension de certains acteurs à s'appuyer sur ce processus pour développer des stratégies financières spécifiques. Celles-ci sont, en effet, davantage susceptibles de diffusion dès lors qu'elles peuvent s'appuyer sur des valeurs (produits ou surtout services) dont le contenu ou la forme glisse du matériel à l'immatériel et de l'immatériel au virtuel. II réside moins, enfin, dans l'accroissement des échanges associé à la mondialisation que dans la capacité de certains acteurs à s'émanciper des 'contraintes' qu'impose la recherche de compromis entre capital et travail au sein d'un même espace (celui de la formation sociale).

Ces acteurs prétendent aujourd'hui constituer un 'bloc social' dominant. II s'agit à l'évidence des acteurs de la finance mondialisée, lesquels se recrutent d'abord sur les marchés financiers (fonds spéculatifs notamment) mais aussi dans des métiers plus 'traditionnels' comme la banque et l'assurance. On songe ici aux comportements adoptés par certains établissements tels que la Deutsche Bank, Allianz, ou encore WestLB, qui, ces dernières années, ont cherché à s'émanciper de leurs liens jusqu'alors privilégiés avec les entreprises et, plus largement, les autres institutions du capitalisme allemand.

II s'agit également d'un certain nombre de grands groupes, au premier rang desquels Daimler Benz, qui ont aussi cherché, avec un succès incertain, à 's'internationaliser', presque toujours par le choix d'une croissance externe marquée par des acquisitions et aussi des cessions (voir, par exemple, la cession controversée au fonds américain KKR de MTU, fournisseur de l'Eurofighter).

II s'agit, enfin, de ceux que R. Reich (L'économie mondialisée, 1993) désigne comme les «manipulateurs de symboles »: cadres dirigeants et autres managers le plus souvent très sensibles aux thèses du capitalisme libéral et à celle de la «gouvernance » d'entreprises, professions libérales et autres détenteurs d'un savoir qualifié contestant une pression fiscale jugée excessive et menaçant de «voter avec leurs pieds », intellectuels convaincus des bienfaits d'une «mondialisation heureuse » ou du moins « non coupable ».

Le commun dénominateur de ce bloc social est la croyance que l'Allemagne devrait s'adapter à la mondialisation et que cette adaptation est irrémédiablement synonyme de contestation et de dépassement du modèle social pensé au lendemain de la guerre (en réalité largement fondé à l'époque bismarckienne). Si, au sein de ce bloc, le principe général de l'économie sociale de marché est rarement, comme tel, remis en cause, il n'est néanmoins plus présenté comme une spécificité dont l'Allemagne tirerait un avantage. Au-delà, tel ou tel aspect de ce système social (telle composante du rapport salarial, comme le niveau élevé des salaires, reflet du niveau de productivité) ou telle ou telle modalité (la recherche de consensus et de compromis négociés notamment) de ce même système social, font l'objet de critiques en ce qu'ils sont supposés être des obs- 
tacles à une mondialisation « réussie », ici le plus souvent désignée par le terme de "globalisation ». A la différence de la mondialisation, qui relève avant tout du jeu d'espaces de plus en plus dépendants ou communicants du fait de la mobilité des facteurs, la globalisation est l'affaire des firmes. Ainsi, si la firme 'multinationale' est la firme représentative de l'internationalisation, la firme 'globale' est celle qui 'pense' au niveau mondial (et agit au niveau local).

Or si ce bloc social exerce une influence considérable depuis une dizaine d'années, il n'est néanmoins pas hégémonique et ne devrait pas réussir à le devenir. Les raisons sont à rechercher ailleurs que dans les cas, assez nombreux au demeurant, d'illustrations d'échecs de 'stratégies' dites mondiales ou globales (Daimler en Asie et même aux Etats-Unis, ou Mannesmann et la dispersion de son capital de contrôle qui a facilité l'OPA de Vodafone, etc.).

La fragilité et l'instabilité extrêmes de l'économie américaine (irrégularité spectaculaire des principaux paramètres économiques et financiers en dépit d'un rythme de croissance élevé, déficits extérieurs considérables, déficits intérieurs croissants), ainsi que la faillite ou du moins les limites évidentes du modèle de capitalisme financiarisé révélées en 2001, mettent en exergue l'impasse que constitue une forme d'idéologie ayant cherché à ériger un modèle de capitalisme prétendant faire abstraction des rapports sociaux de production.

Si le capitalisme financier peut, dans un contexte de déréglementation, prétendre être volatile, il ne peut en aller de même du fonctionnement des capitaux engagés dans la production. Ceux-ci, comme nous l'avons vu, sont tout au plus 'nomades'. Et encore faut-il ici ne pas se tromper sur le sens de ce nomadisme : il se fonde moins sur l'exploitation des écarts de coûts salariaux (les fameuses mais limitées « délocalisations ») que sur la quête de marchés dynamiques et de compétences. C'est ceci qui explique que, si les pays dits développés sont à l'origine des investissements directs, ils en sont aussi, avec certains pays dits émergents, les principaux destinataires.

\section{Le capitalisme allemand: un capitalisme original et qui devrait à l'avenir évoluer plus que se transformer}

Le capitalisme allemand a plus de chance que d'autres capitalismes nationaux de ne pas épouser les contours d'un capitalisme financiarisé. Nous voyons au moins cinq raisons qui constituent autant d'éléments d'ancrage qui devraient empêcher que ne se produise une telle 'dérive'.

La première de ces raisons est le poids relatif de l'industrie dans les activités productives, et l'économie en général. Ce poids est suffisamment important pour que l'Allemagne n'ait de cesse de rappeler la nécessité d'une politique industrielle et de champions industriels européens.

La seconde est que le système productif allemand existe bien comme 'système' et non comme simple somme d'activités ou de secteurs. De nombreux travaux attestent de l'existence de multiples réseaux associant entre elles les entreprises, et les entreprises aux autres acteurs du système productif (acteurs de la recherche et du système de formation, en particulier).

La troisième raison est que si le système bancaire allemand a pu être tenté luimême par une certaine financiarisation de son comportement, c'est-à-dire en l'occurrence l'inversion du lien entre finance et investissement (des investissements choisis en fonction de leur capacité à atteindre une norme de rentabilité financière plutôt que la recherche de la meilleure combinaison de financements pour financer un investissement donné), les 'dérives' observées restent, au final, plutôt limitées. Les banques allemandes demeurent, pour l'essentiel, des partenaires stables, y compris comme actionnaires.

La quatrième raison, proche de la précédente, est que le contrôle du capital des grandes entreprises, à la différence de ce qui est observé en France, reste un
Mais ce bloc social ne fait pas l'opinion car...

... le capitalisme ne peut faire abstraction des rapports sociaux...

...ni d'un certain ancrage des capitaux productifs

Pas de financiarisation du capitalisme rhénan : ...

... le poids de l'industrie...

... une organisation en 'système' productif..

... la stabilité du partenariat banques / entreprises..

... un contrôle 'résident' du capital des entreprises... 
... et le poids des valeurs "rhénanes » en Europe contrôle 'résident', défini donc par des normes produites par des acteurs allemands, même si ceux-ci ne peuvent ignorer les normes en vigueur dans d'autres espaces. De ce point de vue, il nous semble intéressant d'observer que même si la dimension financière de la mondialisation est celle qui fait le moins de doutes, elle ne saurait faire oublier les différences très importantes qui existent d'une zone à l'autre dans la combinaison des trois taux qui 'comptent' (taux d'intérêt, taux de rendement des marchés financiers, taux de change).

La cinquième raison est que l'économie allemande est une économie qui, par son poids et ses valeurs, a la capacité d'influencer le discours et les pratiques au niveau européen. La 'position' allemande, qu'il s'agisse du débat sur l'application ou l'interprétation du Pacte de stabilité, d'une possible initiative commune pour la croissance en Europe, de l'utilité ou de la nécessité d'une harmonisation sur la fiscalité des entreprises dans l'Europe élargie, de la réglementation sur les OPA (etc.), ne peut être ignorée comme cela a pu être observé ces derniers mois. Or ce poids et les valeurs qui constituent le fondement de la société et de l'économie allemande placent le travail - et non la rente - en leur centre.

Au FINAL, LE VÉRITABLE ENJEU pour la société et l'économie allemandes est le glissement progressif vers l'économie de la connaissance - une économie qui se construit au-delà du couplage entre économies d'échelle (production de masse) et économies de variété (production flexible). II s'agit désormais de la capacité que peuvent avoir les entreprises de combiner des compétences et savoirs potentiellement complémentaires afin de résoudre des problèmes 'inédits'. Cette capacité dépend non seulement de la qualification de leurs salariés, mais aussi, voire surtout, de leur aptitude à travailler en équipe, à prendre des initiatives, à être créatifs, qualités ignorées ou combattues par 'l'organisation scientifique du travail'. De ce point de vue, si certaines craintes ont pu à juste titre être exprimées quant à l'évolution récente de l'attention portée aux dépenses d'éducation et de formation et, dans une moindre mesure, de recherche, force est de constater que les exportations allemandes continuent de faire de ce pays un des pays les plus compétitifs au monde, sa compétitivité étant expliquée par ses avantages hors prix. Ces avantages sont à la fois ceux d'une main-d'œuvre particulièrement qualifiée, susceptible de travailler dans des process non standardisables et donc non délocalisables, et ceux qui résultent de comportements individuels et collectifs recherchant à produire fiabilité des engagements et confiance, c'est-à-dire des traits ayant peu à voir avec l'opportunisme et le 'courttermisme' du fonctionnement actuel des marchés financiers. Au final, le modèle rhénan a encore, selon nous, de beaux jours devant lui et pourrait - ou devrait inspirer le modèle social européen.

\section{Indications bibliographiques}

Commissariat GÉnÉRAL du PLAN, La nouvelle nationalité de l'entreprise, La Documentation Française, 1999, et Mondialisation et recomposition du capital des entreprises européennes, La Documentation Française, 2004

DREYFus F.-G., L'aventure du capitalisme rhénan, Clio, octobre 2002

LALLEMENT R., Investissement direct et compétitivité internationale: le cas du système allemand de production et d'innovation, thèse en cours, Toulouse 1

"Le système bancaire allemand face à la mondialisation : vers la fin d'un modèle ? ", Journée d'étude organisée par le CIERA et le CIRAC, Université de Cergy-Pontoise, 12 mars 2004, synthèse reproduite in Regards sur l'Economie allemande, n66/2004

LIST F., Système national d'économie politique, $1^{\text {ère }}$ édition 1841, réédité, Gallimard, 1998 UTERWEDDE H., «Feu le "capitalisme rhénan" ? », Regards sur l'Economie allemande, n55/2002, et intervention dans le cadre d'un débat organisé le 2-10-2003 par le CIRAC : "Quel avenir pour les régimes de protection sociale en Europe ? ", synthèse publiée in Regards sur l'Economie allemande, n64/2003

REICH R., L'économie mondialisée, Economica, 1993

SCHRÖDER G., «Le courage de réformer, l'envie d'innover », Le Monde, 14 mai 2004

StREeCK W., Les capitalismes en Europe, La Découverte, 1996 